\title{
Morte Súbita em Epilepsia - O que é preciso saber?
}

\section{Sudden Death in Epilepsy - What do we need to know?}

\author{
Fernando Netto Zanette', Guilherme Loureiro Fialho², Katia Lin
}

1 Acadêmico do Curso de Graduação em Medicina, Centro de Ciências da Saúde, Universidade Federal de Santa Catarina, Campus Reitor João David Ferreira Lima, Florianópolis

2. Médico do Hospital Universitário Prof. Polydoro Ernani de São Thiago, Universidade Federal de Santa Catarina, Campus Reitor João David Ferreira Lima, Florianópolis

3 Professora do Departamento de Clínica Médica, Centro de Ciências da Saúde, Universidade Federal de Santa

Catarina, Campus Reitor João David Ferreira Lima, Florianópolis

Artigo submetido em 23/11/2018

Aceito para publicação em 19/12/2018

Autor para contato: Fernando Netto Zanette (zanettefernando@gmail.com)

DOI: https://doi.org/10.32963/bcmufsc.v4i12.3308

\section{RESUMO}

Introdução: A Morte Súbita Inesperada na Epilepsia - Sudden Unexpected Death in Epilepsy (SUDEP) - define-se como morte em pacientes com epilepsia (PCE), associada ou não a convulsão, de forma súbita, inesperada, presenciada ou não, excluindo-se causas traumáticas, afogamento e estado de mal epiléptico, onde a necropsia não revele origem toxicológica ou anatômica do óbito. Objetivo: Apresentar uma revisão narrativa, não-sistemática, das informações mais importantes e recentes sobre SUDEP, destacando definição, fatores de risco, diagnóstico e medidas preventivas com o intuito de auxiliar médicos na orientação e identificação desses pacientes em risco. Metodologia: Foi realizada extensa revisão em bases de dados eletrônicos com pesquisa em Cochrane, PubMed/Medline, BMJ e Scielo, tendo como critério de inclusão artigos de grande relevância, publicados em português ou inglês. Resultados/Discussão: A SUDEP pode afetar indivíduos de todas as idades, sendo mais prevalente em adultos jovens (20-45 anos). A presença e severidade de crises tônico-clônico generalizadas (CTCGs) são os fatores de risco mais relevantes. Sua fisiopatologia envolve modificações autonômicas progressivas com deterioração do sistema cardiorrespiratório. $A$ medida preventiva mais incentivada é o adequado controle das crises epilépticas. Conclusão: Muitos PCE nunca ouviram falar na SUDEP, o que se deve, diversas vezes, ao receio ou dificuldade de médicos em mencioná-la aos indivíduos que não estão sob alto risco do evento. 0 médico tem papel fundamental na educação desses pacientes, para garantir a informação sobre a epilepsia e tentar minimizar os fatores de risco associados à SUDEP e, consequentemente, seus efeitos deletérios na vida desses indivíduos.

Palavras-chave: Epilepsia, SUDEP, Morte Prematura, Convulsão, Crise epiléptica

\section{ABSTRACT}

Introduction: Sudden Unexpected Death in Epilepsy (SUDEP) is defined as death of patients with epilepsy (PWE), associated or not with a seizure, in a sudden and unexpected way, witnessed or unwitnessed, excluding traumatic, drowning and documented status epilepticus, in which post-mortem examination does not reveal a toxicological or anatomical cause of death. Objective: It aims to 
present a non-systematic narrative review of the most important and recent information about SUDEP, highlighting definition, risk factors, diagnosis and preventive measures in order to assist physicians in counseling and identification of these patients at risk. Methodology: An extensive review was conducted in electronic databases with a Cochrane, PubMed/Medline, BMJ and Scielo search, with inclusion criteria being articles of high relevance published in Portuguese or English. Results/Discussion: SUDEP can affect individuals of all ages, but with greater prevalence in young adults (20-45 years old). The presence and severity of generalized tonic-clonic seizures (GTCSs) stand out as the most relevant risk factors behind SUDEP. The pathophysiology involves progressive autonomic modifications with deterioration of the cardiorespiratory system. The most encouraged preventive measure is adequate management of epileptic seizures. Conclusion: Many PWEs have never heard of SUDEP, which is often due to the fear or difficulty to mention it to individuals who are not at high risk for the event. The physician plays a key role in educating these patients, to ensure information about epilepsy and try to minimize the risk factors associated with SUDEP and, consequently, its deleterious effects on the lives of these individuals.

Keywords: Epilepsy, SUDEP, Premature Death, Seizure, Epileptic Seizure

\section{INTRODUÇÃO}

A epilepsia se destaca como uma das afecções neurológicas mais comuns na prática clínica, afetando cerca de 65 milhões de pessoas, de todas as idades e gêneros, em âmbito global ${ }^{1,2,3}$. A despeito de sua tratabilidade, é uma doença de curso crônico, necessitando, muitas vezes, de uso de medicamentos por toda a vida e, em alguns casos, até mesmo abordagens neurocirúrgicas para 0 controle das crises ${ }^{4,5}$. Aproximadamente $40 \%$ dos pacientes com epilepsia (PCE), no entanto, não respondem de forma satisfatória às drogas antiepilépticas (DAEs) ou a outras alternativas ${ }^{6}$. Nesses indivíduos com epilepsia refratária, altas taxas de morte prematura, em comparação à população geral e aos PCE responsivos ao tratamento, são encontradas ${ }^{7,8}$.

A presença da epilepsia, por si, já acarreta uma elevação de 2 a 3 vezes no risco global de mortalidade pelas diversas causas $^{9-18}$ - como, por exemplo, status epiléptico, acidentes automobilísticos, quedas, afogamento, suicídio, intoxicação alimentar, assaltos e pneumonia 19,20. As crises epilépticas, no entanto, - especialmente as focais ou as crises tônico-clônico generalizadas (CTCGs) - são as causas mais recorrentes de óbito em PCE 20,21,22, com a morte súbita inesperada nas epilepsias (Sudden Unexpected Death in Epilepsy [SUDEP]) tendo a maior relevância (Quadro 1).

Quadro 1 - Morte relacionada à epilepsia

Individuos com epilepsia possuem um risco 2 a 3 vezes superior de morte em comparação à população saudável.

Fonte: Adaptado de THURMAN, et al. (2016)
A mortalidade prematura associada à epilepsia impõe um fardo significante no sistema público de saúde dos diversos países, e muitas vezes é potencialmente prevenível. Esse dado exige atenção de profissionais de saúde e pesquisadores. ${ }^{9}$

Levando-se em consideração o grande impacto da SUDEP, a epilepsia não deve ser considerada uma condição benigna, o que demanda do médico adequada orientação aos seus pacientes e manejo correto das crises epilépticas.

\section{OBJETIVO}

0 artigo tem por objetivo apresentar uma revisão narrativa, não-sistemática, das informações mais importantes e recentes acerca da SUDEP, destacando-se definição, classificação, fatores de risco, diagnóstico e medidas preventivas com o intuito de auxiliar médicos - generalistas ou especialistas - na orientação e identificação desses pacientes em risco.

\section{MÉTODO}

Como procedimento metodológico, foi realizada uma extensa revisão em bases de dados eletrônicos com pesquisa em Cochrane, PubMed/Medline, BMJ e Scielo, abrangendo artigos publicados nas línguas portuguesa e inglesa com palavras-chave "epilepsia", "SUDEP", "morte prematura", "convulsão", "crise convulsiva" "epilepsy", "premature death", "seizure" e "epileptic seizure". Os critérios de inclusão se definiam como artigos destacados como de grande relevância (classes 1-4). A partir da leitura atenta e interpretativa, excluíram-se os artigos duplicados e que não se adequaram aos objetivos do trabalho. Foi utilizado um total de 103 artigos publicados que tinham como objetivo caracterizar a epilepsia e a SUDEP, destacando fatores de risco, causas, prevalência e prevenção.

\section{RESULTADOS / DISCUSSÃO}




\section{Sudden unexpected death in epilepsy (SUDEP)}

A Morte Súbita Inesperada em Epilepsia (SUDEP) é definida como a morte em pacientes com epilepsia, associada ou não a uma convulsão, de forma súbita, inesperada, presenciada ou não, excluindo-se causas traumáticas, afogamento e estado de mal epiléptico, onde a necropsia não revele uma origem toxicológica ou anatômica por trás do óbito23 - a exemplo de morte por acidente vascular encefálico (AVE), infarto agudo do miocárdio (IAM) ou intoxicação por drogas. Com base em sua definição, tem-se a SUDEP como um diagnóstico de exclusão. Um resumo sobre os princípios básicos da SUDEP pode ser encontrado no Quadro 2.

\section{Quadro 2 - Sintese dos fatos sobre SUDEP}

\section{Definição}

Define-se como uma morte súbita, inesperada, não traumática e não relacionada a afogamento de um individuo com epilepsia, presenciada ou não, na qual a necrópsia não revele uma causa anatômica ou toxicológica por trás do óbito.

\section{Incidência}

O risco de morte súbita em pessoas com epilepsia é cerca de 27 vezes superior à população saudável de mesma faixa etária.

A incidência de SUDEP pode ser estimada em $1 / 10.000$ paciente ano para novos casos de epilepsia; 1-2/1.000 paciente/ano para casos crônicos de epilepsia; e 2-10/1.000 pacientes/ano para casos de epilepsia refratária ao tratamento. Em crianças, as taxas são inferiores às outras faixas etárias, de $0,2 / 1.000$ paciente/ano.

\section{Causas}

Com diversos mecanismos propostos para a entidade, não existe uma explicação universalmente aceita. A imensa maioria dos casos ocorre após uma convulsão tônicoclônico generalizada (CTCG). Os casos presenciados e estudados apontam para uma disfunção cardiorrespiratória pós-ictal. Dentre os fatores de risco propostos, um histórico de CTCG se destaca como o mais importante.

Fonte: Adaptado de SHANKAR, et al. (2017)

\section{Epidemiologia}

A incidência precisa da SUDEP ainda não é totalmente estabelecida, provavelmente em virtude de sua subnotificação ou da dificuldade de diversos médicos de a reconhecerem, ou, muitas vezes, terem pouco conhecimento sobre a entidade.

Estudos apontam que a morte súbita nas epilepsias se enquadra como a causa mais frequente de mortes relacionadas à epilepsia ${ }^{7,24-}$ ${ }^{26}$, responsabilizando-se por $17 \%$ de todos esses óbitos $1,27,28$, com uma taxa de incidência de 1/500-1/1.000 adultos com epilepsia/ano e uma média de 0,2/1.000 crianças com epilepsia/ano 1,27,29.
A SUDEP possui uma incidência em adultos jovens (idade entre 2045 anos) 27 vezes superior à morte súbita na população geral de mesma idade ${ }^{30}$. Juntamente a isso, nos EUA, a SUDEP se qualifica como segunda maior causa neurológica de potencial de anos de vida perdido, atrás apenas do AVE ${ }^{31}$.

Após o pico de incidência em jovens adultos, a SUDEP se reduz progressivamente com 0 avanço da idade, especialmente acima dos 60 anos, como pode ser visto representado na Figura 1. A entidade, no entanto, pode ser subdiagnosticada em adultos mais velhos em que a morte súbita, muitas vezes, termina por ser atribuída a desfechos cardíacos (como, por exemplo, IAM) sem investigações adicionais de causas alternativas ${ }^{20}$. 


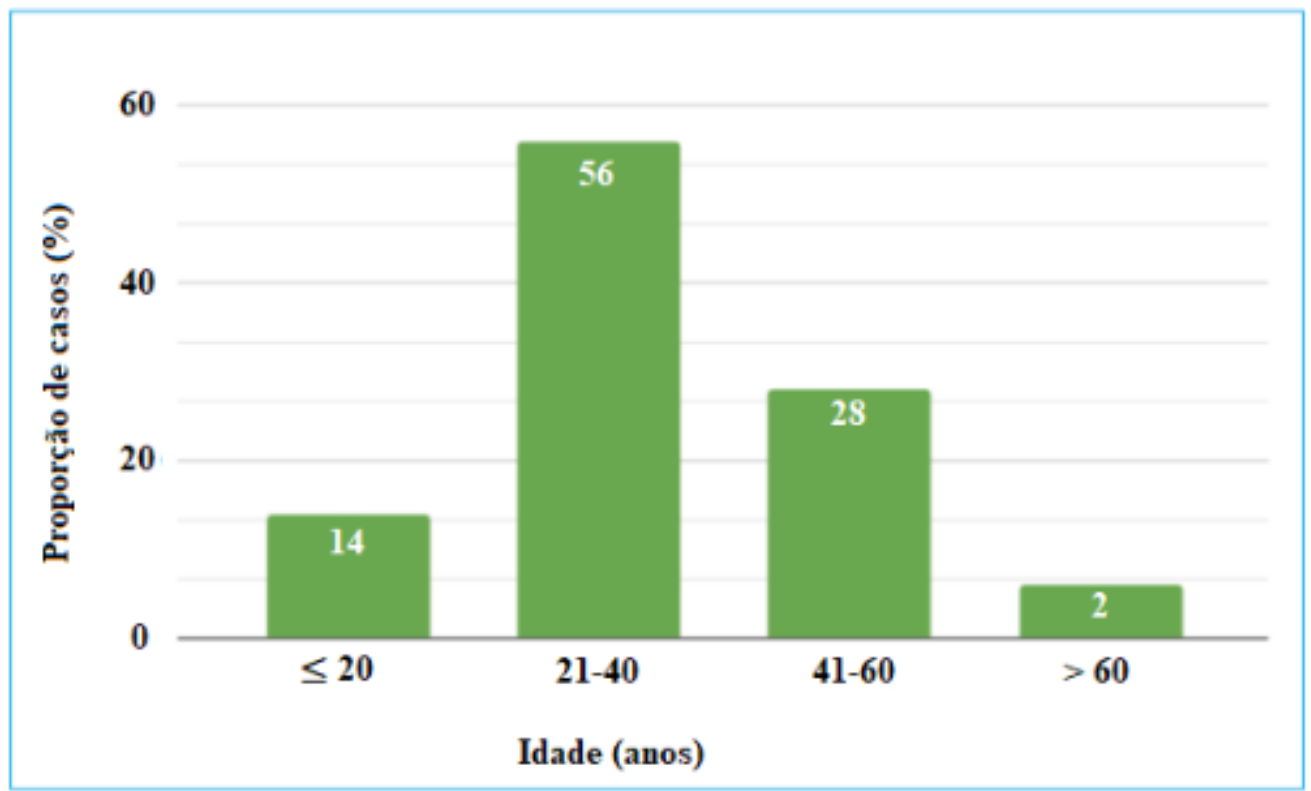

Figura 1 - Distribuição de casos de SUDEP de acordo com a idade

Fonte: Adaptado de DEVINSKY, et al. (2016)

\section{Fatores de risco}

Dentre os principais fatores de risco da SUDEP, os mais relevantes são: presença de CTCGs, frequência anual das crises e crises refratárias ao tratamento farmacológico ou cirúrgico. Um resumo dos dados pode ser encontrado no Quadro 3.

Quadro 3 - Principais fatores de risco referentes à SUDEP

\begin{tabular}{|cc|}
\hline $\begin{array}{c}\text { Presença de convulsões tônico-clônico } \\
\text { generalizadas }\end{array}$ & $\begin{array}{c}\text { Idade jovem }(<16 \text { anos) ao início } \\
\text { da doença epiléptica }\end{array}$ \\
$\begin{array}{c}\text { Frequência anual das convulsões } \\
\text { Não adesão à terapia anticonvulsiva } \\
\text { ou epilepsia refratária ao tratamento }\end{array}$ & Sindrome de Dravet (crianças) \\
& Gênero masculino \\
\hline
\end{tabular}

Fonte: Produzido pelo autor

\section{Tipo de epilepsia}

De todos os fatores de risco, aqueles com maior peso são: a presença de CTCGs e seu controle inadequado 20,32-45 - seja pela falta de adesão ou resistência ao tratamento. No caso da refratariedade, no entanto, a probabilidade da SUDEP se eleva, com um risco absoluto de 1 a cada 150 pacientes/ano ${ }^{35}$.

\section{Frequência anual das crises}

A frequência anual das CTCGs, por sua vez, aumenta em até 5 vezes a probabilidade do desfecho em pacientes com um a dois episódios dessas crises anualmente, saltando para até 15 vezes nos casos de três ou mais crises anuais $20,24,33,42-46$. Esse incremento no risco relativo se traduz para um risco absoluto de até 18 mortes a cada 1.000 pacientes/ano para pessoas com CTCGs frequentes ( $\geq 3$ crises/ano $)^{34,46}$.

\section{Idade de início da doença}

A ocorrência de SUDEP se correlaciona de forma inversamente proporcional à idade de início das crises epilépticas. Idade jovem à primeira crise epiléptica $20,32-37$, antes dos 16 anos, amplifica em até 1,72 vez a probabilidade de sua ocorrência.

\section{Tempo de duração da doença}

Doença de longa data também afeta o prognóstico desses indivíduos. Os pacientes com tempo de doença superior a 15 anos possuem um risco até 1,95 vez superior quando comparado à população saudável de mesma faixa etária20,32-37.

\section{Crises no período noturno}

A SUDEP acontece durante o período de sono (04h-08h da madrugada) em $58,5 \%$ dos casos 20,47 , e as convulsões noturnas apresentam um aumento de 2,6 vezes na possibilidade do desfecho - após o ajuste adequado de outros fatores de risco (como número total de DAEs utilizadas) ${ }^{48}$. Por outro lado, a companhia de 
alguém com idade superior a 10 anos ou a checagem frequente durante a noite/uso de aparelhos monitorizadores de som reduziram o risco de SUDEP em 2,5 e 10 vezes, respectivamente ${ }^{32}$.

\section{Outros fatores}

A não adesão ao tratamento, privação do sono, apneia do sono não tratada, abuso ou abstinência em etilistas, estresse, ciclo menstrual e outros podem intensificar, de forma combinada ou isolada, a probabilidade de ocorrência de crises epilépticas em determinados grupos de pacientes. Uma vez que a frequência das crises se associa à SUDEP, tais componentes são referidos como possíveis potencializadores dessa entidade 20,33 .

Outros fatores de risco propostos incluem gênero masculino, deficiência cognitiva, número das DAEs, uso de lamotrigina (em mulheres), ausência de doenças cerebrovasculares, asma, etiologia sintomática da epilepsia (causa estrutural/metabólica) e baixas temperaturas24,28,34,37-,39,49-51.

Nas crianças, a presença da Síndrome de Dravet ou Epilepsia Mioclônica Grave da Infância (EMGI) está especialmente associada a um incremento na ocorrência de SUDEP 28,33.

\section{Fisiopatologia}

O mecanismo fisiopatológico subjacente da SUDEP ainda permanece desconhecido. Todavia, as principais hipóteses incluem desregulação da função autonômica acompanhada de alterações cardiovasculares e respiratórias $28,37,38,52-58$, isoladas ou combinadas, durante e após as crises epilépticas (período ictal e pós-ictal, respectivamente).

Esse evento ocorre usualmente na fase pós-ictal de uma CTCG, com uma cascata de disfunção respiratória ${ }^{22}$, falha no estímulo, disfunção cardíaca de caráter não-taquiarritmogênico (por exemplo, bradicardia ou bigeminismo ou ritmo irregular), e supressão generalizada da atividade elétrica cerebral pós-ictal conhecida como depressão cortical profunda da atividade eletroencefalográfica ${ }^{59}$. Existem, todavia, relatos de morte súbita nos pacientes por disfunção cardíaca de caráter taquiarritmogênico - como fibrilação ventricular ou taquicardia ventricular. 60

O desfecho dessa via fisiopatológica se traduz no grave comprometimento da função cardiopulmonar mediada centralmente decorrente da convulsão, com uma apneia terminal normalmente precedendo assistolia e óbito $28,33,61-63$.

\section{Estado pós-ictal}

Após a CTCG, temos uma redução no estímulo e atividade cerebral, com ativação predominante dos componentes simpáticos do sistema nervoso 20,64,65. Essa descarga simpática, a qual aumenta os níveis de adrenalina sérica e do líquido cerebrospinal por até mais de 30 minutos após a crise ${ }^{64}$, pode se manifestar na forma de hipertermia, taquicardia, hipertensão, atividade eletrodérmica e edema pulmonar. De fato, observa-se edema pulmonar de moderado a severo nos pacientes com SUDEP à necrópsia28,42,66-68.

Há também uma excessiva descarga parassimpática na SUDEP, sugerindo que o estímulo ictal/pós-ictal tanto do ramo simpático

Na Figura 2 tem-se a representação da fisiopatologia SUDEP, a qual ocorre após uma crise tônico-clônico generalizada (número 1) em um indivíduo suscetível (número 2). Potencializadores de risco (número 3) e consequências diretas das crises (número 4) interagem de forma heterogênea com desfecho, provavelmente devido a uma combinação de hipoventilação e hipóxia, arritmia ou uma combinação de ambas.

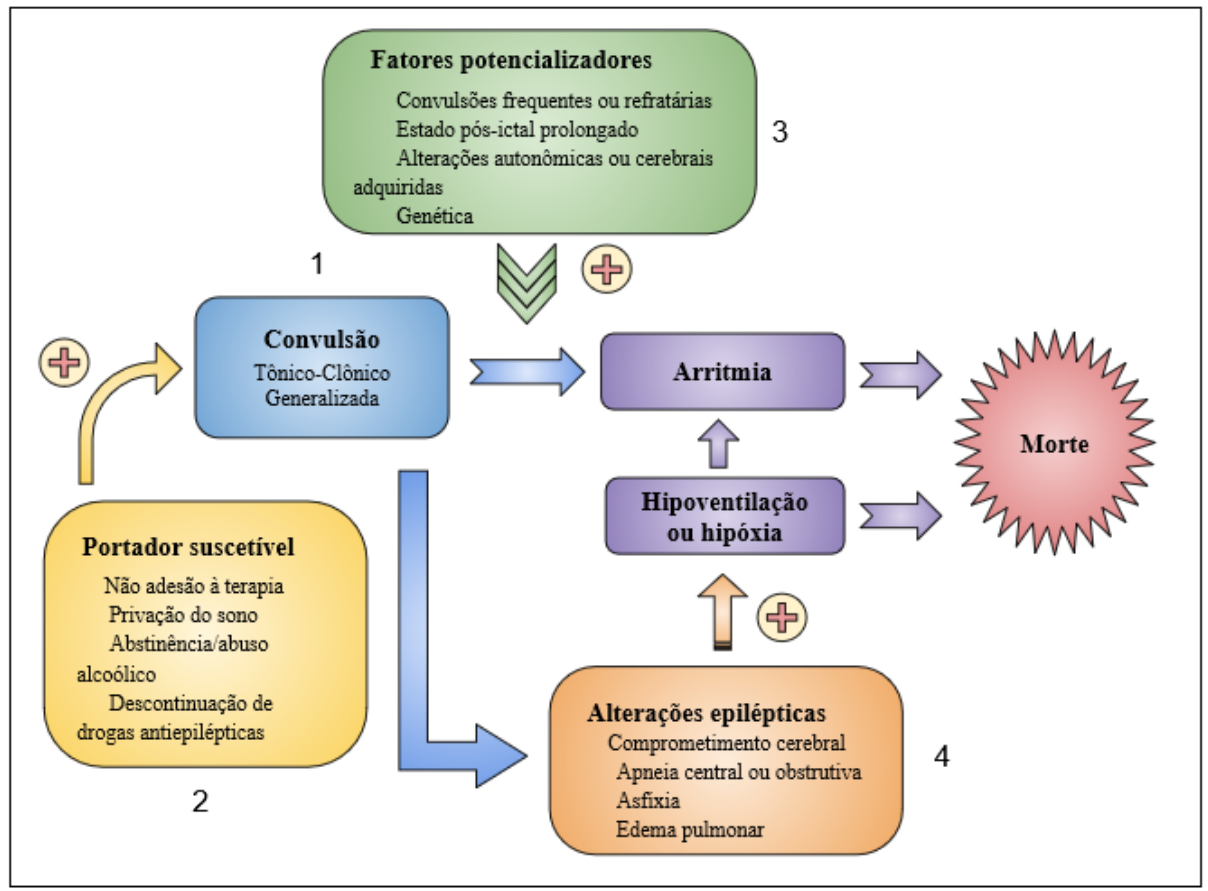

Fonte: Produzido pelo autor

Figura 2 - Modelo da fisiopatologia da SUDEP 


\section{Função cardiorrespiratória e controle autonômico}

Durante as crises epilépticas, episódios de hipoventilação e apneia tanto obstrutiva quanto central são recorrentes ${ }^{69,70}$ - às vezes associados a desfechos altamente fatais nos modelos animais $69,71-75$-, reforçando a hipótese de que a apneia ictal/pósictal é um contribuinte importante para a SUDEP. A redução na capacidade de oxigenação, além disso, acarreta uma hipercapnia pós-ictal, podendo levar a quadros de acidose grave, altamente arritmogênicos.

Os quadros convulsivos, juntamente a isso, implicam em modificações importantes na dinâmica cardíaca que podem estar relacionadas à ocorrência de SUDEP. Nos modelos animais, a cronicidade e a elevada frequência das crises epilépticas se relacionam a um aumento da pressão arterial média, da frequência cardíaca em repouso e da susceptibilidade a arritmias induzidas por isquemia e reperfusão28,76-80. É possível que essa disautonomia crônica predisponha a uma fibrose miocárdica e seja um substrato anatômico arrítmico para a morte súbita60.

\section{A neurotransmissão serotoninérgica}

Dentre os diversos neurotransmissores envolvidos no controle respiratório, a serotonina se encontra associada à modulação das respostas à hipóxia e ao despertar induzido pela hipercapnia ( $\uparrow$ [CO2] no sangue arterial), além de ser relacionada à modulação das convulsões ${ }^{81-85}$. Com base nisso, o crescente número de estudos sugere que defeitos genéticos ou adquiridos no sistema serotoninérgico podem predispor o paciente ao desfecho da SUDEP28.

\section{Susceptibilidade}

Não se sabe ao certo, no entanto, por que a SUDEP ocorre em alguns pacientes após poucas crises epilépticas, ao passo que outros são poupados independentemente de uma vida de CTCGs. Predisposição genética, embora não concretamente estudada, pode estar relacionada, uma vez que mutações nos genes de canais iônicos expressados nos tecidos cerebral e cardíaco podem acarretar uma susceptibilidade aumentada a crises epilépticas, disfunção autonômica do tronco encefálico e $\operatorname{arritmias}^{57,86,87}$.

\section{Prevenção}

A prevenção da SUDEP só pode ser alcançada por meio de um esforço combinado de neurologistas, epileptologistas, médicos generalistas, epidemiologistas, sistema público de saúde e pacientes. Apesar do crescente número de estudos nos últimos anos acerca da SUDEP, a incerteza concreta de seus mecanismos fisiopatológicos nos restringe de forma considerável no que diz respeito ao seu adequado manejo e prevenção. As medidas preventivas disponíveis são direcionadas aos fatores de risco potencialmente modificáveis.

\section{Controle das Crises Epilépticas}

Uma vez que a frequência das CTCGs é o principal fator de risco da SUDEP, fica claro que o adequado manejo das crises é indispensável para sua prevenção $33,34,88$, de forma proativa, incluindo otimização do tratamento e encaminhamento a serviços especializados ${ }^{33}$. Em indivíduos com epilepsia refratária, por exemplo, a terapia otimizada em doses adequadas de DAEs pode reduzir em até 7 vezes o desfecho da SUDEP quando comparado ao grupo placebo 33,88 .

Em casos onde o uso de duas ou mais DAEs é insuficiente para controlar as crises, o diagnóstico de epilepsia deve ser reavaliado, ao mesmo tempo em que é ponderada a possibilidade de tratamento cirúrgico ${ }^{35}$. A abordagem neurocirúrgica, quando realizada nos pacientes com adequada indicação e quando bem-sucedida, tende a reduzir consideravelmente o risco de SUDEP 89

\section{Educação}

Devemos lembrar que o paciente desempenha um papel central na prevenção da SUDEP, sendo fundamental a sua adequada instrução por parte dos médicos. Dada a importância no controle das convulsões para a SUDEP, o aconselhamento ao PCE deve incluir uma discussão sobre seus fatores desencadeadores como álcool, privação do sono e, principalmente, a não adesão ao tratamento -, e a importância em como evitá-los de forma individualizada.

Embora seja temida por alguns neurologistas e médicos generalistas, a conversa sobre a epilepsia e seus potenciais riscos - destacando-se a SUDEP - com o paciente é uma etapa indispensável no processo, sendo benéfica na maioria das situações ${ }^{56,90}$. Menos de $15 \%$ dos PCE, seus parentes e seu cuidadores recebem informações sistemáticas em relação à SUDEP e à mortalidade associada à epilepsia21,33,91-94. A Tabela 1 aborda uma sugestão de conversa sobre a SUDEP em uma pessoa recém-diagnosticada com epilepsia.

Tabela 1. Sugestão de abordagem ao paciente recémdiagnosticado com epilepsia

1. Planeje uma consulta antecipada para discutir com seu paciente o diagnóstico recente de epilepsia e suas possíveis complicações. Ofereça a ele, caso interessado, trazer um membro da família ou amigo próximo.

2. Crie um ambiente confortável e dê tempo ao paciente para tirar possíveis dúvidas.

3. Pergunte se eles já ouviram falar no termo SUDEP ou morte súbita na epilepsia e se teriam interesse em saber mais do assunto.

4. Explique a entidade, de maneira compreensível, focando nos fatores de risco e na associação com a crise epiléptica. Reforce a raridade da SUDEP e correlacione com outros riscos do dia a dia, como ser atropelado ou assaltado.

5. Destaque medidas que podem auxiliar o paciente a reduzir as crises e o risco da SUDEP, como adesão medicamentosa e remoção de fatores de risco individuais.

6. Permita ao paciente tempo para refletir sobre a conversa, mantendo-se disponível para esclarecimento de novas dúvidas durante a consulta ou em abordagens futuras.

Fonte: Adaptado de SHANKAR et al. (2017) 


\section{Quando Falar Sobre a SUDEP?}

Para muitos médicos, pode ser difícil determinar 0 momento apropriado para tratar da SUDEP com PCE e seus familiares ${ }^{95}$. As diretrizes do National Institute for Health and Care Excellence (NICE) e do Scottish Intercollegiate Guidelines Network (SIGN) advogam que a discussão da SUDEP com o paciente deve ser feita o quanto antes possível|95,96 pelo médico encarregado do caso do paciente ou, quando apropriado, por um enfermeiro especializado em epilepsia. Alguns estudos vêm defendendo que a imensa maioria dos PCE, seus parentes e/ou seus cuidadores (quando apropriado) devem ser advertidos sobre o risco da SUDEP já no momento do diagnóstico ${ }^{95,97}$. A conversa sobre o desfecho só deve ser evitada em casos selecionados nos quais exista a possibilidade de dano ao paciente ao prover tal informação ${ }^{97}$.

Deve-se ter em mente que, no entanto, após serem informadas acerca de um evento adverso, as pessoas usualmente superestimam a probabilidade de tal desfecho ocorrer a elas ${ }^{98}, 0$ que pode ser minimizado ao se adicionar números além de palavras $^{98}$ e a frequência dos riscos em vez de percentagens ${ }^{99}$.

\section{Outras Medidas}

Pela SUDEP ocorrer com maior frequência no período da madrugada e nas primeiras horas da manhã, os estudos apontam para um efeito protetor de dividir o quarto do paciente com alguém capaz de prover assistência durante uma crise convulsiva ${ }^{32}$, indicando que ao menos alguns riscos associados com convulsões noturnas sejam relacionados à falta de supervisão ${ }^{100}$. Há um mercado em rápida expansão de aparelhos de detecção e alerta de convulsões. No entanto, a eficácia de tais aparelhos ainda permanece incerta 32,33

O uso de medicamentos neuromoduladores, em especial dos inibidores seletivos de recaptação de serotonina (ISRS), apresentam certo efeito protetor em reduzir a dessaturação e a hipóxia em humanos ${ }^{28,101}$. Além disso, fármacos que aumentam a atividade serotoninérgica (como os ISRS) e agonistas da serotonina diminuem a suscetibilidade a convulsões tanto em modelos animais ${ }^{81,102}$ quanto em humanos ${ }^{103}$. Isso reforça a hipótese de que o manejo da disfunção serotoninérgica pode ser uma medida alternativa na tentativa de prevenir a SUDEP, já que a serotonina desempenha um papel fundamental não apenas no controle respiratório e no despertar, mas também no controle das convulsões 28 .

\section{CONCLUSÃO}

A epilepsia, uma das afecções neurológicas mais frequentes na prática médica, é importante causador de estigmas, morbidade e mortalidade entre seus portadores, sendo a SUDEP a causa mais comum de óbito em PCE. Embora possa acometer indivíduos de todas as idades, sua maior ocorrência se faz em adultos jovens (idade entre 20-45 anos), com uma incidência média de 1/1.000 casos/ano em PCE crônica. Dentre os principais fatores de risco da SUDEP, a presença e a severidade de CTCGs são os com maior destaque clínico sendo, usualmente, alvos de possíveis medidas preventivas.

Apesar de ainda não totalmente esclarecida, a sua fisiopatologia envolve modificações autonômicas progressivas pela ocorrência frequente de convulsões - com consequente deterioração/sobrecarga dos sistemas cardiovascular e respiratório. Frequentemente, tem-se um quadro de crise convulsiva severa, seguida por alterações a nível cerebral do sistema de regulação cardiovascular, hipóxia, apneia, arritmia e óbito.

Muitas medidas preventivas vêm sendo estudadas e propostas, porém, em virtude de sua fisiopatologia, as mais fortemente incentivadas dizem respeito ao adequado controle das crises epilépticas, especialmente as CTCGs. A instrução adequada aos PCE, relembrando fatores desencadeantes - como álcool e não adesão medicamentosa - e como evitá-los de forma individualizada, também é um passo importante na prevenção da SUDEP.

Apesar de sua prevalência e do importante impacto à saúde que causa, muitos PCE e seus familiares nunca ouviram falar na SUDEP. Isso se deve, em diversas situações, ao receio ou dificuldade de médicos - até mesmo neurologistas especializados em epilepsia - em mencioná-la aos pacientes que não estão sob alto risco para o evento, apreensivos do impacto que a informação causará. A conversa adequada e antecipada acerca da epilepsia e da SUDEP com o PCE, todavia, é medida a ser incentivada, visto os efeitos benéficos, de forma geral, que essa abordagem traz no entendimento e possibilidade de melhora do prognóstico dos pacientes.

O médico, juntamente à sua equipe multidisciplinar, tem um papel fundamental na educação desses pacientes e seus familiares, não somente para garantir de maneira precisa a informação sobre a epilepsia, mas também tentar minimizar os fatores de risco associados à SUDEP. A articulação conjunta entre profissionais de saúde e pacientes é um de muitos passos essenciais em direção à possibilidade de prevenção desse desfecho tão devastador que, infelizmente, ceifa a vida de incontáveis indivíduos.

\section{Referências}

1. Scorza FA, Esper A.C., Costa, J.C. Sudden cardiac death in epilepsy disappoints, but epileptologists keep faith. Arq Neuropsiquiatr 2016;74(7):570-573. DOI: 10.1590/0004$282 \times 20160086$

2. Banerjee PN, Filippi D, Hauser WA. The descriptive epidemiology of epilepsy: a review. Epilepsy Res. 2009:85(1):31-45. doi:10.1016/i.eplepsyres.2009.03.003

3. Moshé SL, Perucca E, Ryvlin P, Tomson T. Epilepsy: new advances. Lancet. 2015;385(9971):884-98. doi:10.1016/S0140-6736(14)60456-6

4. Forsgren I, Beghi E, Ekman M. Cost of epilepsy in Europe. Eur J Neurol. 2005;12(Suppl s1):54-8. doi:10.1111/j.1468-1331.2005. 01191.x

5. Allers K, Essue BM, Hackett ML, Muhunthan J, Anderson CS, Pickles K et al. The economic impact of epilepsy: a systematic review. BMC Neurol. 2015;15(1):245. doi:10.1186/s12883-015-0494-y

6. Laxer KD, Trinka E, Hirsch LJ, Cendes F, Langfitt J, Delanty N et al. The consequences of refractory epilepsy and its treatment. Epilepsy Behav. 2014; 37:59-70. doi:10.1016/j.yebeh.2014.05.031 
7. Nashef L, Ryvlin P. Sudden unexpected death in epilepsy (SUDEP): update and reflections. Neurol Clin. 2009;27(4):1063-74. doi:10.1016/j.ncl.2009.08.003

8. Duncan JS, Sander JW, Sisodiya SM, Walker MC. Adult epilepsy. Lancet. 2006; 367(9516):1087-100. doi:10.1016/S0140-6736(06)68477-8

9. David J. Thurman, Logroscino G, Beghi E., Hauser W.A., Hesdorffer D.C., Newton C.R., et al. The burden of premature mortality of epilepsy in high-income countries: A systematic review from the Mortality Task Force of the International League Against Epilepsy. Epilepsia, ${ }^{* *}\left({ }^{\star}\right): 1-10,2016$ doi: 10.1111/epi.13604

10. Cockerell OC, Johnson AL, Sander JW, et al. Prognosis of epilepsy: a review and further analysis of the first nine years of the British National General Practice Study of Epilepsy, a prospective population-based study. Epilepsia 1997; 38:31-46.

11. Neligan A, Bell GS, Johnson AL, et al. The long-term risk of premature mortality in people with epilepsy. Brain 2011; 134:388-395.

12. Benn EKT, Hauser WA, Shih T, et al. Estimating the incidence of first unprovoked seizure and newly diagnosed epilepsy in the low-income urban community of Northern Manhattan, New York City. Epilepsia 2008; 49:1431-1439.

13. Hauser WA, Annegers JF, Elveback LR. Mortality in patients with epilepsy. Epilepsia 1980; 21:399-412.

14. Lhatoo SD, Johnson AL, Goodridge DM, et al. Mortality in epilepsy in the first 11 to 14 years after diagnosis: multivariate analysis of a long-term, prospective, population-based cohort. Ann Neurol 2001; 49:336-344.

15. Lindsten H, Nystrom L, Forsgren L. Mortality risk in an adult cohort with a newly diagnosed unprovoked epileptic seizure: a population based study. Epilepsia 2000; 41:14691473 .

16. Morgan CL, Kerr MP. Epilepsy and mortality: a record linkage study in a U.K. population. Epilepsia 2002; 43:1251-1255.

17. Olafsson E, Hauser WA, Gudmundsson G. Long-term survival of people with unprovoked seizures: a population-based study. Epilepsia 1998; 39:89-92.

18. Rakitin A, Liik M, Oun A, et al. Mortality risk in adults with newly diagnosed and chronic epilepsy: a population-based study. Eur J Neurol 2011; 18:465-470

19. Fazel S, Wolf A, Langstrom N, Newton CR, Lichtenstein P. Premature mortality in epilepsy and the role of psychiatric comorbidity: a total population study. Lancet 2013; 382: 1646-54.

20. Devinsky O, Hesdorffer, D.C., Thurman, D.J., Lhatoo, S., Richerson, G. Sudden unexpected death in epilepsy: epidemiology, mechanisms, and prevention. The Lancet Neurology.

Vol 15 September 2016; 1075-1088.

21. Devinsky O, Spruill T, Thurman D, Friedman D. Recognizing and preventing epilepsy-related mortality: a call for action. Neurology 2016; 86: 779-86.

22. Massey CA, Sowers LP, Dlouhy BJ, Richerson GB. Mechanisms of sudden unexpected death in epilepsy: the pathway to prevention. Nat Rev Neurol 2014; 10: 271-82.

23. Nashef L. Sudden unexpected death in epilepsy: terminology and definitions. Epilepsia. 1997;38(Suppl s11):S6-8. doi:10.1111/j.1528-1157. $1997 . t b 06130$.

24. Hesdorffer DC, Tomson T, Benn E, et al. Combined analysis of risk factors for SUDEP. Epilepsia. 52(6), 1150-9 (2011).

25. Nei M, Hays R. Sudden unexpected death in epilepsy. Curr. Neurol. Neurosci. Rep. 10(4), 319-26 (2010).

26. Sander J, Bell G. Reducing mortality: an important aim of epilepsy management. J Neurol Neurosurg Psychiatry 2004; 75: 349-51

27. Schuele SU, Widdess-Walsh P, Bermeo A, Lüders HO. Sudden unexplained death in epilepsy: the role of the heart. Cleve Clin J Med. 2007;74(Suppl 1):S121-7. doi:10.3949/ccjm.74.Suppl_1. S121

28. Pansani, A.P., Colugnati, D.B., Scorza, C.A., Almeida, A.C.G, Cavalheiro, E.A., Scorza, F.A. Furthering our understanding of SUDEP: the role of animal models. Expert Review of Neurotherapeutics, DOI: 10.1586/14737175.2016.1169925

29. Donner EJ, Smith CR, Snead OC 3rd. Sudden unexplained death in children with epilepsy. Neurology. 2001;57(3):430-4. doi:10.1212/WNL.57.3.430

30. Holst AG, Winkel BG, Risgaard B, et al. Epilepsy and risk of death and sudden unexpected death in the young: a nationwide study. Epilepsia 2013; 54: 1613-20.

31. Thurman DJ, Hesdor! er DC, French JA. Sudden unexpected death in epilepsy: assessing the public health burden. Epilepsia 2014; 55: 1479-85.

32. Langan Y, Nashef L, Sander JW. Case-control study of SUDEP. Neurology 2005; 64: 1131-33.

33. Shankar, R., Donner, E.J., McLean, B., Nashef, L., Tomson, T. Sudden unexpected death in epilepsy (SUDEP): what every neurologist should know. Epileptic Disord, Vol. 19,

No. 1, March 2017

34. Harden, C., Tomson, T., Gloss, D., Buchhalter, J., Cross, J. H., Donner, E., et al. Practice guideline summary: Sudden unexpected death in epilepsy incidence rates and risk factors. Report of the Guideline Development, Dissemination, and Implementation Subcommittee of the American Academy of Neurology and the American Epilepsy Society. 2017 American Academy of Neurology

35. So, E.L., Brainbridge, J., Buchhalter, J.R., Donalty, J., Donner, E.J., Finucane, A., et al. Report of the American Epilepsy Society and the Epilepsy Foundation Joint Task Force on Sudden Unexplained Death in Epilepsy. Epilepsia, 50(4):917-922, 2009 doi: 10.1111/j.1528-1167.2008.01906.

36. Scorza FA, et al, SUDEP: A steep increase in publication since its definition, Epilepsy Behav (2017), http://dx.doi.org/10.1016/.jyebeh.2017.05.006

37. Devinsky O. Sudden, unexpected death in epilepsy. N Engl J Med. 2011;365(19):1801-11.

38. Tomson T, Nashef L, Ryvlin P. Sudden unexpected death in epilepsy: current knowledge and future directions. Lancet Neurol 2008; 7:1021-31

39. Surges R, Thijs R, Tan H, et al. Sudden unexpected death in epilepsy: risk factors and potential pathomechanisms. Nat Rev Neurol 2009; 5: 492-504.

40. Tomson T, Surges R, Delamont R, et al. Who to target in sudden unexpected death in epilepsy prevention and how? Risk factors, biomarkers, and intervention study designs. Epilepsia 2016; 57(1): 4-16.

41. Shankar R, Walker M, McLean B, Laugharne, R., Ferrand, F., Hanna, J., et al. Steps to prevent SUDEP: the validity of risk factors in the SUDEP and seizure safety checklist: a case control study. J Neurol 2016; 263(9): 1840-6.

42. Tomson T, Walczak T, Sillanpaa M, Sander JW. Sudden unexpected death in epilepsy: a review of incidence and risk factors. Epilepsia 2005;46:Suppl 11:54-61.

43. Nilsson L, Farahmand BY, Persson PG, Thiblin I, Tomson T. Risk factors for sudden unexpected death in epilepsy: a casecontrol study. Lancet 1999; 353:888-93.

44. Opeskin K, Berkovic SF. Risk factors for sudden unexpected death in epilepsy: a controlled prospective study based on coroners cases. Seizure 2003; 12:456-64.

45. Sevcencu C, Struijk JJ. Autonomic alterations and cardiac changes in epilepsy. Epilepsia 2010; 51:725-37.

46. Hesdorffer DC, Tomson T, Benn E, Sander JW, Nilsson L, Langan Y, et al. Commission on epidemiology. Subcommission on mortality combined analysis of risk factors for SUDEP. Epilepsia 2011; 52(6): 1150-9.

47. Lamberts RJ, Laranjo S, Kalitzin SN, Velis DN, Rocha I., Sander JW, et al. Postictal generalized EEG suppression is not associated with periictal cardiac autonomic instability in people with convulsive seizures. Epilepsia 2013; 54: 523-29.

48. Lamberts RJ, Thijs RD, La! an A Langan Y, Sander JW. Sudden unexpected death in epilepsy: people with nocturnal seizures may be at highest risk. Epilepsia 2012; 53: 25357 .

49. Hesdorffer DC, Tomson T. Sudden unexpected death in epilepsy: potential role of antiepileptic drugs. CNS Drugs. 2013;27(2):113-9

50. Hitiris N, Suratman S, Kelly K, Stephen LJ, Sills GJ, Brodie MJ. Sudden unexpected death in epilepsy: a search for risk factors. Epilepsy Behav. 2007;10(1):138-41

51. Scorza FA, Terra VC, Arida RM, Scorza CA, Cavalheiro EA. Sudden unexpected death in epilepsy and winter temperatures: it's important to know that it's c-c-c-ccold outside. Epilepsy Behav. 14(4), 707; author reply 708 (2009).

52. Stöllberger C, Finsterer J. Cardiorespiratory findings in sudden unexplained/unexpected death in epilepsy (SUDEP). Epilepsy Res. 59(1), 51-60 (2004).

53. Seyal M, Pascual F, Lee C-YM, Li C-S, Bateman LM. Seizure-related cardiac repolarization abnormalities are associated with ictal hypoxemia. Epilepsia. 52(11), 2105-2111 (2011).

54. Seyal M, Bateman LM, Li CS. Impact of periictal interventions on respiratory dysfunction, postictal EEG suppression, and postictal immobility. Epilepsia. 54(2), 377-382 (2013).

55. van der Lende M, Surges R, Sander JW, Thijs RD. Cardiac arrhythmias during or after epileptic seizures. J. Neurol. Neurosurg. Psychiatry. 1-6 (2015).

56. Surges R, Sander JW. Sudden unexpected death in epilepsy: mechanisms, prevalence, and prevention. Curr. Opin. Neurol. 25(2), 201-7 (2012).

57. Goldman AM, Behr ER, Semsarian C, Bagnall RD, Sisodiya S, Cooper PN. Sudden unexpected death in epilepsy genetics: molecular diagnostics and prevention. Epilepsia. 2016;57 Suppl 1:17-25.doi:10.1111/epi.13232 
58. Goldman AM. Mechanisms of sudden unexplained death in epilepsy. Curr Opin Neurol. 2015;28(2):166-74. doi:10.1097/WCO.0000000000000184

59. Lhatoo SD, Faulkner HJ, Dembny K, Trippick K, Johnson C, Bird JM. An electroclinical case-control study of sudden unexpected death in epilepsy. Ann Neurol 2010; 68: 787-96.

60. Fialho GL, Wolf P, Walz R, Lin K. Increased cardiac stiffness is associated with autonomic dysfunction in patients with temporal lobe epilepsy. Epilepsia. 2018 Jun; 59(6):e85-90.

61. Ryvlin P, Nashef L, Lhatoo S, et al. Incidence and mechanisms of cardiorespiratory arrests in epilepsy monitoring units (MORTEMUS): a retrospective study. Lancet Neurol 2013; 12: $966-77$.

62. Blum AS. Respiratory physiology of seizures. J. Clin. Neurophysiol. 26(5), 309-15 (2009).

63. Scorza FA, de Albuquerque R, Arida RM, Schmidt B, de Almeida AC, Scorza CA, et al. Could sudden death syndrome (SDS) in chickens (Gallus) be a valid animal model for sudden unexpected death in epilepsy (SUDEP)? Med. Hypotheses. 73(1), 67-9 (2009).

64. Simon RP. Heart and lung in the postictal state. Epilepsy Behav 2010; 19: 167-71.

65. Sarkis RA, Thome-Souza S, Poh MZ, Llewellyn N, Klehm J, Madsen JR, et al. Autonomic changes following generalized tonic clonic seizures: An analysis of adult and pediatric patients with epilepsy.

66. P-Codrea Tigaran S, Dalager-Pedersen S, Baandrup U, Dam M, Vesterby-Charles A. Sudden unexpected death in epilepsy: is death by seizures a cardiac disease? Am J Forensic Med Pathol 2005; 26: 99-105.

67. Johnston SC, Horn JK, Valente J, Simon RP. The role of hypoventilation in a sheep model of epileptic sudden death. Ann. Neurol. 37(4), 531-7 (1995).

68. Surges R, Adjei P, Kallis C, Erhuero J, Scott CA, Bell GS, et al. Pathologic cardiac repolarization in pharmacoresistant epilepsy and its potential role in sudden unexpected death in epilepsy: a case-control study. Epilepsia. 51(2), 233-42 (2010).

69. St.-John WM, Rudkin AH, Homes GL, Leiter JC. Changes in respiratorymodulated neural activities, consistent with obstructive and central apnea, during fictive seizures in an in situ anaesthetized rat preparation. Epilepsy Res. 70, 218- 228 (2006).

70. Bateman LM, Li CS, Seyal M. Ictal hypoxemia in localization-related epilepsy:analysis of incidence, severity and risk factors. Brain 2008; 131:3239-45

71. Johnston SC, Siedenberg R, Min JK, Jerome EH, Laxer KD. Central apnea and acute cardiac ischemia in a sheep model of epileptic sudden death. Ann. Neurol. 42(4), 588-94 (1997).

72. Faingold CL, Randall M, Tupal S. DBA/1 mice exhibit chronic susceptibility to audiogenic seizures followed by sudden death associated with respiratory arrest. Epilepsy Behav. 17(4), 436-440 (2010).

73. Kalume F, Westenbroek RE, Cheah CS, Yu FH, Oakley JC, Scheuer T, et al. Sudden unexpected death in a mouse model of Dravet syndrome. J. Clin. Invest. 123(4), 1798-808 (2013).

74. Moore BM, Jerry Jou C, Tatalovic M, Kaufman ES, Kline DD, Kunze DL. The Kv1.1 null mouse, a model of sudden unexpected death in epilepsy (SUDEP). Epilepsia. 55(11), 1808-1816 (2014).

75. Aiba I, Noebels JL. Spreading depolarization in the brainstem mediates sudden cardiorespiratory arrest in mouse SUDEP models. Sci. Transl. Med. 7, 282ra46 (2015)

76. Lathers CM, Schraeder PL. Autonomic dysfunction in epilepsy: characterization of autonomic cardiac neural discharge associated with pentylenetetrazol-induced epileptogenic activity. Epilepsia. 23(6), 633-47 (1982)

77. Goodman JH, Homan RW, Crawford IL. Kindled seizures elevate blood pressure and induce cardiac arrhythmias. Epilepsia. 31(5), 489-95 (1990).

78. Colugnati DB, Gomes PAP, Arida RM, et al. Analysis of cardiac parameters in animals with epilepsy: possible cause of sudden death? Arq. Neuropsiquiatr. 63(4), 1035-41 (2005).

79. Damasceno DD, Ferreira AJ, Doretto MC, Almeida AP. Cardiovascular dysautonomia after seizures induced by maximal electroshock in Wistar rats. Seizure. 21(9), 711-6 (2012).

80. Damasceno DD, Savergnini SQ, Gomes ERM, Guatimosim S, Ferreira AJ, Doretto MC, et al. Cardiac dysfunction in rats prone to audiogenic epileptic seizures. Seizure. 22(4),

259-66 (2013)

81. Buchanan GF, Smith HR, MacAskill A, Richerson GB. 5-HT2A receptor activation is necessary for CO2-induced arousal. J. Neurophysiol. 114(1):233:43 (2015).

82. Buchanan GF, Murray NM, Hajek M a., Richerson GB. Serotonin neurones have anti-convulsant effects and reduce seizure-induced mortality. J. Physiol. .592(19), 4395-4410 (2014).

83. Richerson GB, Buchanan GF. The serotonin axis: Shared mechanisms in seizures, depression, and SUDEP. Epilepsia. 52 Suppl 1, 28-38 (2011).

84. Richerson GB. Serotonergic neurons as carbon dioxide sensors that maintain pH homeostasis. Nat. Rev. Neurosci. 5(6), 449-61 (2004).

85. Feldman JL, Mitchell GS, Nattie EE. Breathing: rhythmicity, plasticity, chemosensitivity. Annu. Rev. Neurosci. 26, 239-66 (2003).

86. Johnson JN, Hofman N, Haglund CM, Cascino GD, Wilde AA, Ackerman MJ. Identification of a possible pathogenic link between congenital long QT syndrome and epilepsy. Neurology 2009; 72:224-31.

87. Nashef L, Sander JW. Genes cut across systems: neurologists should think "heart" and cardiologists "brain". Neurology 2016; 87(16): 1638-9.

88. Ryvlin P, Cucherat M, Rheims S. Risk of sudden unexpected death in epilepsy in patients given adjunctive antiepileptic treatment for refractory seizures: a meta-analysis of placebo-controlled randomised trials. Lancet Neurol 2011; 10: 961-68.

89. Sperling M, Harris A, Nei M, Liporace JD, O'Connor MJ. Mortality after epilepsy surgery. Epilepsia 2005; 46: 49-53.

90. Amorin, HA, Scorza, CA, Cavalheiro, EA, de Albuquerque, M., Scorza, F.A., et al. Profile of neurologists in Brazil: a glimpse into the future of epilepsy and sudden unexpected death in epilepsy. Neurologists in Brazil and the future of epilepsy. CLINICS 2013;68(7):896-898

91. Kroner BL, Wright C, Friedman D, Macher K, Preiss L, Misajon J, et al. Characteristics of epilepsy patients and caregivers who either have or have not heard of SUDEP. Epilepsia 2014; 55: 1486-94.

92. Xu Z, Ayyappan S, Seneviratne U. Sudden unexpected death in epilepsy (SUDEP): what do patients think? Epilepsy Behav 2015; 42:29-34.

93. Gayatri N, Morrall M, Jain V, et al. Parental and physician beliefs regarding the provision and content of written sudden unexpected death in epilepsy (SUDEP) information. Epilepsia 2010; 51: 777-82.

94. Ramachandrannair R, Jack S, Meaney B, et al. SUDEP: what do parents want to know? Epilepsy Behav 2013; 29: 560-4.

95. Shankar R., Cox D, Jalihal V, Brown S, Hanna J, McLean B. Sudden unexpected death in epilepsy (SUDEP): Development of a safety checklist. Seizure 22 (2013) $812-817$

96. Clinical Guideline 137 NICE. Partial pharmacological update of clinical guideline 20: the epilepsies: the diagnosis and management of the epilepsies in adults and children in primary and secondary care. London: National Clinical Guideline Centre; 2012 January: 22.

97. Nashef L, Leach JP. SUDEP, the aftermath: supporting the bereaved. Pract Neurol 2017; 0:1-4. doi:10.1136/practneurol-2017-001729

98. Knapp P, Raynor DK, Berry DC. Comparison of two methods of presenting risk information to patients about the side effects of medicine. Qual Saf Health Care 2004; 13:176180.

99. Bonner C, Newell BR. How to make a risk seem riskier: the ratio bias versus construal level theory. Judgment Decis Making 2008; 3:411-416.

100. Sander J. Reducing the risk of sudden death during epilepsy monitoring. Lancet Neurol 2013; 12(10): 935-6.

101. Bateman LM, Li CS, Lin TC, Seyal M. Serotonin reuptake inhibitors are associated with reduced severity of ictal hypoxemia in medically refractory partial epilepsy. Epilepsia. 51(10), 2211-2214 (2010).

102. Wada Y, Shiraishi J, Nakamura M, Hasegawa H. Prolonged but not acute fluoxetine administration produces its inhibitory effect on hippocampal seizures in rats. Psychopharmacology (Berl). 118(3), 305-9 (1995).

103. Favale E, Rubino V, Mainardi P, Lunardi G, Albano C. Anticonvulsant effect of fluoxetine in humans. Neurology. 45(10), 1926-7 (1995). 


\section{Sobre o "Boletim do Curso de Medicina da UFSC"}

\section{Submissões para publicação:}

Preferencialmente online através do sistema Open Journals System. Acesso em: http://ojs.sites.ufsc.br/index.php/medicina

Crie seu login e senha para acesso à revista através da página de cadastro, clicando em "Sobre" e em "Submissões"

\section{Diretrizes para Autores}

Trabalhos enviados para publicação devem ser montados em arquivo .doc ou .docx, em língua portuguesa, com a seguinte estrutura:

Página de rosto: indicando a sessão em que pretende publicar o trabalho (veja detalhes das sessões em "Políticas de Sessão"), título, identificação dos autores em ordem de citação (nome completo, setor de trabalho, titulação e email de contato), endereço para correspondência do autor responsável pelos contatos.

A declaração de conflitos de interesse dos autores deve aparecer na folha de rosto e será publicada junto ao trabalho. Incluir fontes de apoio financeiro ao trabalho e citar se os autores possuem vinculação a empresas/coletivos/partidos que tenham interesse na divulgação das informações do trabalho.

Deve ser anexado um documento .pdf contendo o seguinte texto: "Declaração de concordância - os autores abaixo assinados declaram que concordam com a publicação do trabalho intitulado (escrever o título do trabalho) em "Boletim do Curso de Medicina da UFSC" e que aceitam as normas de publicação neste periódico. Os autores declaram os seguintes conflitos de interesse: (declarar ligação a empresas/coletivos/partidos/movimentos com interesse na divulgação das informações do trabalho). Cidade, data - Nome completo, e-mail e assinatura de todos os autores"

Segunda página (esta, em português e em inglês): Resumo com até 250 palavras (estruturado da mesma forma que 0 artigo), seguido de três a seis palavras-chave, separadas por ponto-e-vírgula, para indexação (baseadas no DECs - descritores em ciências da saúde)

Em seguida, o artigo (com o máximo de 3000 palavras). Se artigo original, estruturado em seções (Introdução, Objetivos, Método, Resultados, Discussão e Conclusões). Artigos de revisão narrativa podem ter estrutura Introdução, Método, Revisão e Conclusões. As sessões "Artigos", "o que o residente lê" e "Cartas" tem estrutura livre e não exigem resumo.

Trabalhos envolvendo pesquisa com seres humanos ou animais devem mencionar a aprovação no respectivo Comitê de Ética em Pesquisa. Ensaios clínicos devem mencionar o registro em órgão competente.

O artigo deve ser escrito em fonte Times New Roman, tamanho 12, espaço simples. As referências devem seguir a norma de Vancouver, conforme divulgadas nas orientações para o Trabalho de Conclusão de Curso da Medicina (http://www.medicina.ufsc.br/files/2011/11/NORMAS-TCC-MEDUFSC-2011.pdf) e nas orientações de normatização de trabalhos acadêmicos da Biblioteca Universitária (http://portal.bu.ufsc.br/normalizacao/), sendo numeradas ao longo do texto em algarismos arábicos sobrescritos.

Figuras e tabelas devem seguir as mesmas normas do TCC da medicina (http://www.medicina.ufsc.br/files/2011/11/NORMAS-TCC-MEDUFSC-2011.pdf). Tabelas podem ser incorporadas no mesmo arquivo do texto; figuras podem ser incorporadas no texto, mas também devem ser encaminhadas como "arquivo suplementar". 